DOI: $10.17516 / 1997-1397-2021-14-1-28-41$

УДК 517.9

\title{
On a Limiting Passage as the Thickness of a Rigid Inclusions in an Equilibrium Problem for a Kirchhoff-Love Plate with a Crack
}

\author{
Nyurgun P. Lazarev* \\ Galina M. Semenova ${ }^{\dagger}$ \\ Natalya A. Romanova ${ }^{\ddagger}$ \\ North-Eastern Federal University \\ Yakutsk, Russian Federation
}

\begin{abstract}
Received 10.05.2020, received in revised form 10.07.2020, accepted 20.09.2020
Abstract. The paper considers equilibrium models of Kirchhoff-Love plates with rigid inclusions of two types. The first type of inclusion is described by three-dimensional sets, the second one corresponds to a cylindrical rigid inclusion, which is perpendicular to the plate's median plane in the initial state. For both models, we suppose that there is a through crack along a fixed part of the inclusion's boundary. On the crack non-penetration conditions are prescribed which correspond to a certain known configuration bending near the crack. The uniqueness solvability of a new problems for a Kirchhoff-Love plate with a flat rigid inclusion is proved. It is proved that when a thickness parameter tends to zero, the problem for a flat rigid inclusion can be represented as a limiting task for a family of variational problems concerning the inclusions of the first type. A solvability of an optimal control problem with a control given by the size of inclusions is proved.
\end{abstract}

Keywords: variational problem, crack, limit passage, nonpenetration condition, optimal control problem.

Citation: N.P. Lazarev, G.M. Semenova, N.A. Romanova, On a Limiting Passage as the Thickness of a Rigid Inclusions in an Equilibrium Problem for a Kirchhoff-Love Plate with a Crack, J. Sib. Fed. Univ. Math. Phys., 2021, 14(1), 28-41. DOI: 10.17516/1997-1397-2021-14-1-28-41.

\section{Introduction}

It is well known that presence of inhomogeneities such as cracks, holes and inclusions in loaded bodies can cause local high stress concentrations near these objects. The features of mechanical properties and geometric parameters of inclusions can affect the initiation and propagation of a crack. At present, active studies of problems for various models of elastic bodies containing rigid inclusions and cracks with both linear and nonlinear boundary conditions are being carried out, and we refer the reader, for example, to [1-11]. Using the universality of the methods of the calculus of variations, various problems for bodies with rigid inclusions have been successfully formulated and investigated, see, for example, [1,12-21]. In particular, within the framework of two-dimensional problems of elasticity theory, the first mathematical model on the equilibrium of

\footnotetext{
*nyurgun@ngs.ru https://orcid.org/0000-0002-7726-6742

${ }^{\dagger}$ sgm08@yandex.ru

${ }^{\ddagger}$ nan.romanova@s-vfu.ru

(C) Siberian Federal University. All rights reserved
} 
a body with nonlinear Signorini-type conditions on a part of the boundary of a thin delaminated rigid inclusion was proposed in [1]. The three-dimensional case is considered in [22]. The work [23] is devoted to the analysis of the shapes of cracks and rigid inclusions in elastic bodies. A formula for the derivative of an energy functional with respect to the perturbation parameter of the domain in an equilibrium problem for an elastic body with a thin delaminated inclusion was obtained in [14]. For Kirchhoff-Love plates containing a thin rigid inclusion, the cases with both delamination (i.e. with a crack) and a solid plate without a crack were studied in [19]. We should note that, in this work, for solid plates without delamination of inclusions, it was established that, passing to the limit in a family of equilibrium problems describing plates with a volume inclusion, when the transverse size of the inclusions tends to zero, an equilibrium problem for a plate with a thin inclusion is obtained as the limit one.

In this article, two types of equilibrium problems for a Kirchhoff-Love plate with a rigid inclusion and a flat rigid inclusion are investigated. More precisely, volume inclusions are defined by three-dimensional domains and flat inclusions are given by cylindrical surfaces. Unlike [19], deformation of a cylindrical rigid inclusion is modeled in such a way that rotation angles of normal fibers of the plate are equal to some constant values within the entire plane rigid inclusion in equilibrium state. For all cases, it is assumed that there is a crack is at the inclusion's boundary. This study investigates the effect of changing the transverse thickness of the inclusion for the considered mathematical model. An optimal control problem is formulated with a quality functional characterizing the deviation of a solution from a given function. The control is set by a parameter of the transverse thickness of the rigid inclusion. The existence of an optimal inclusion's size is proved. In addition, a qualitative connection has been established between equilibrium problems for Kirchhoff-Love plates with delaminated flat rigid inclusions and problems for delaminated volume inclusions. In particular, the strong convergence of the solutions for problems with volume inclusions to the solution of the problem for a flat inclusion is proved when the parameter of the transverse thickness of the volume inclusion tends to zero. It should be noted that boundary conditions of the inequality type are imposed on the crack for the case of a known bending configuration.

\section{Formulation of a contact problem}

Let us formulate an equilibrium problem for an elastic plate containing a volume rigid inclusion. We consider the case of the delaminated inclusion, when the crack passes through the inclusion interface. Let $\Omega \subset \mathbf{R}^{2}$ be a bounded with a smooth boundary $\Gamma$. Suppose that a smooth unclosed curve $\gamma$ lies strictly inside $\Omega$, i.e. $\bar{\gamma} \subset \Omega$. We require that the curve $\gamma$ can be extended up to the outer boundary $\Gamma$ in such a way that $\Omega$ is divided into two subdomains $\Omega_{1}$, $\Omega_{2}$ with Lipschitz boundaries. The latter condition is sufficient to fulfil the Korn and Poincare inequalities in the domain $\Omega_{\gamma}=\Omega \backslash \bar{\gamma}[24]$. We consider the family of simply-connected domains $\omega_{t} \subset \Omega, t \in\left(0, t_{0}\right]$ with the following properties:

a) the boundaries $\partial \omega_{t}$ are smooth such that $\partial \omega_{t} \in C^{1,1}$;

b) $\omega_{t} \subset \omega_{t^{\prime}}, \bar{\omega}_{t^{\prime}} \subset \Omega$ for all $t, t^{\prime} \in\left(0, t_{0}\right], t<t^{\prime}$;

c) for any fixed $\hat{t} \in\left(0, t_{0}\right)$ and any neighborhood $\mathcal{O}$ of the domain $\bar{\omega}_{t}$ there exists $t_{\mathcal{O}}>\hat{t}$ such that $\omega_{t} \subset \mathcal{O}$ for all $t \in\left[\hat{t}, t_{\mathcal{O}}\right]$;

d) for any neighborhood $\mathcal{O}$ of the curve $\gamma$ there exists $t_{\mathcal{O}}>0$ such that $\omega_{t} \subset \mathcal{O}$ for all $t \in\left(0, t_{\mathcal{O}}\right]$

e) $\gamma \subset \partial \omega_{t}$ for all $t \in\left(0, t_{0}\right]$; 
f) $\bigcup_{t<t^{\prime}} \omega_{t}=\omega_{t^{\prime}}$ for all $t^{\prime} \in\left(0, t_{0}\right]$.

As an example, the family of domains $\omega_{t}\left(\bar{\omega}_{t} \subset \Omega\right), t \in\left(0, t_{0}\right]$ with boundaries $\partial \omega_{t}=\gamma \cup \gamma_{t} \cup \gamma_{t}^{1} \cup \gamma_{t}^{2}$, with

$$
\begin{gathered}
\gamma=\left\{\left(x_{1}, x_{2}\right) \mid-a<x_{1}<0, \quad x_{2}=g\left(x_{1}\right)\right\}, \\
\gamma_{t}=\left\{\left(x_{1}, x_{2}\right) \mid-a<x_{1}<0, \quad x_{2}=g\left(x_{1}\right)-2 t\right\}, \quad g \in C^{1,1}\left[-t_{0}, 1+t_{0}\right]
\end{gathered}
$$

and with semicircles $\gamma_{t}^{1}, \gamma_{t}^{2}$ satisfies properties a) - f) (see Figure 1). For this example the domain's thickness along the $O x_{2}$ axis is equal to $2 t$.

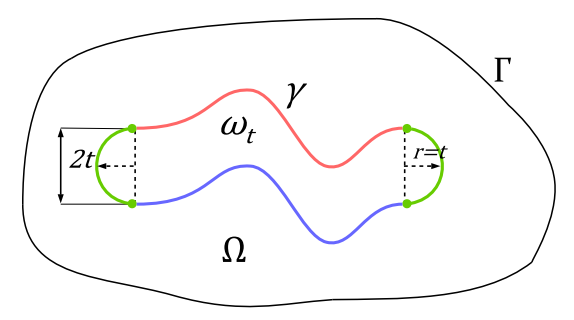

Fig. 1. Example of the domains $\omega_{t}$

For simplicity, suppose the plate has a uniform thickness $2 h=2$. Let us assign a threedimensional Cartesian space $\left\{x_{1}, x_{2}, z\right\}$ with the set $\left\{\Omega_{\gamma}\right\} \times\{0\} \subset \mathbf{R}^{3}$ corresponding to the middle plane of the plate. The curve $\gamma$ defines a crack (a cut) in the plate. This means that the cylindrical surface of the through crack may be defined by the relations $x=\left(x_{1}, x_{2}\right) \in \gamma,-1 \leqslant z \leqslant 1$ where $|z|$ is the distance to the middle plane. For fixed $t \in\left(0, t_{0}\right]$ the volume rigid inclusion is specified by the set $\omega_{t} \times[-1,1]$, i.e. the boundary of the rigid inclusion is defined by the cylindrical surface $\partial \omega_{t} \times[-1,1]$. An elastic part of the plate corresponds to the domain $\Omega \backslash \bar{\omega}_{t}$. Depending on the direction of the normal $\nu=\left(\nu_{1}, \nu_{2}\right)$ to $\gamma$ we will speak about a positive face $\gamma^{+}$or a negative face $\gamma^{-}$of the curve $\gamma$. The jump $[q]$ of the function $q$ on the curve $\gamma$ is found by the formula $[q]=\left.q\right|_{\gamma^{+}}-\left.q\right|_{\gamma^{-}}$.

Denote by $\chi=\chi(x)=(W, w)$ the displacement vector of the mid-surface points $\left(x \in \Omega_{\gamma}\right)$, by $W=\left(w_{1}, w_{2}\right)$ the displacements in the plane $\left\{x_{1}, x_{2}\right\}$, and by $w$ the displacements along the axis $z$. The strain and integrated stress tensors are denoted by $\varepsilon_{i j}=\varepsilon_{i j}(W), \sigma_{i j}=\sigma_{i j}(W)$, respectively [24]:

$$
\varepsilon_{i j}(W)=\frac{1}{2}\left(\frac{\partial w_{j}}{\partial x_{i}}+\frac{\partial w_{i}}{\partial x_{j}}\right), \quad \sigma_{i j}(W)=a_{i j k l} \varepsilon_{k l}(W), \quad i, j=1,2,
$$

where $\left\{a_{i j k l}\right\}$ is the given elasticity tensor, assumed as usual to be symmetric and positive definite:

$$
\begin{array}{r}
a_{i j k l}=a_{k l i j}=a_{j i k l}, \quad i, j, k, l=1,2, \quad a_{i j k l} \in L^{\infty}\left(\Omega_{\gamma}\right), \\
a_{i j k l} \xi_{i j} \xi_{k l} \geqslant c_{0}|\xi|^{2}, \quad \forall \xi, \quad \xi_{i j}=\xi_{j i}, \quad i, j=1,2, \quad c_{0}=\text { const }>0 .
\end{array}
$$

A summation convention over repeated indices is used in the sequel. Next we denote the bending moments by formulas [24]

$$
m_{i j}(w)=d_{i j k l} w, k l, \quad i, j=1,2, \quad\left(w, k l=\frac{\partial^{2} w}{\partial x_{k} \partial x_{l}}\right)
$$


where the tensor $\left\{d_{i j k l}\right\}$ has the same properties as the tensor $\left\{a_{i j k l}\right\}$. Let $B(\cdot, \cdot)$ be a bilinear form defined by the equality

$$
B(\chi, \bar{\chi})=\int_{\Omega_{\gamma}}\left\{\sigma_{i j}(W) \varepsilon_{i j}(\bar{W})+m_{i j}(w) \bar{w},{ }_{i j}\right\} d x,
$$

where $\chi=(W, w), \bar{\chi}=(\bar{W}, \bar{w})$. The potential energy functional of the plate has the following representation [24]:

$$
\Pi(\chi)=\frac{1}{2} B(\chi, \chi)-\int_{\Omega_{\gamma}} F \chi d x, \quad \chi=(W, w),
$$

where vector $F=\left(f_{1}, f_{2}, f_{3}\right) \in L_{2}(\Omega)^{3}$ describes the body forces [24]. Introduce the Sobolev spaces

$$
\begin{gathered}
H^{1,0}\left(\Omega_{\gamma}\right)=\left\{v \in H^{1}\left(\Omega_{\gamma}\right) \mid v=0 \text { on } \Gamma\right\}, \\
H^{2,0}\left(\Omega_{\gamma}\right)=\left\{v \in H^{2}\left(\Omega_{\gamma}\right) \mid v=\frac{\partial v}{\partial n}=0 \text { on } \Gamma\right\}, \\
H\left(\Omega_{\gamma}\right)=H^{1,0}\left(\Omega_{\gamma}\right)^{2} \times H^{2,0}\left(\Omega_{\gamma}\right),
\end{gathered}
$$

where $n=\left(n_{1}, n_{2}\right)$ is the normal vector to $\Gamma$. Note that the following inequality

$$
B(\chi, \chi) \geqslant c\|\chi\|^{2} \quad \forall \chi \in H\left(\Omega_{\gamma}\right), \quad\left(\|\chi\|=\|\chi\|_{H\left(\Omega_{\gamma}\right)}\right)
$$

with a constant $c>0$ independent of $\chi$, holds for the bilinear form $B(\cdot, \cdot)[24]$.

Remark 1. The inequality (2) yields the equivalence of the standard norm and the semi-norm determined by the bilinear form $B(\cdot, \cdot)$ in the space $H\left(\Omega_{\gamma}\right)$.

In the case of prior knowledge that a certain configuration of plate edges near a crack results in an equilibrium state (see Fig. 2), we impose following mutual nonpenetration condition of opposite crack faces [25]

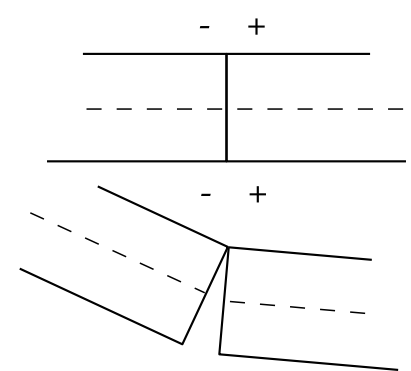

Fig. 2. An example of crack edges configurations for initial (the upper image) and equilibrium (the lower image) states

$$
\left[\frac{\partial w}{\partial \nu}\right] \geqslant 0, \quad[W] \nu \geqslant\left[\frac{\partial w}{\partial \nu}\right], \quad[w]=0 \quad \text { on } \quad \gamma .
$$

In addition, we can mention that if condition (3) holds, then the following general nonpenetration condition for cracks in Kirchhoff-Love plates is fulfilled [21,24].

$$
[W] \nu \geqslant\left|\left[\frac{\partial w}{\partial \nu}\right]\right| \text { on } \gamma
$$


Due to presence of a rigid inclusion in the plate, restrictions of the functions describing displacements $\chi$ to the corresponding domain $\omega_{t}$ satisfy a special kind of relations. We introduce the space which allows us to characterize the properties of the volume rigid inclusion

$$
\begin{aligned}
R\left(\omega_{t}\right)=\{ & \zeta(x)=(\rho, l) \mid \rho(x)= \\
& \left.=b\left(x_{2},-x_{1}\right)+\left(c_{1}, c_{2}\right) ; l(x)=a_{0}+a_{1} x_{1}+a_{2} x_{2}, \quad x \in \omega_{t}\right\},
\end{aligned}
$$

where $b, c_{1}, c_{2}, a_{0}, a_{1}, a_{2} \in \mathbf{R}[21,26]$.

The variational formulation describing the equilibrium state for the elastic plate with the volume delaminated rigid inclusion can be formulated as follows:

$$
\text { find } \xi_{t}=\left(U_{t}, u_{t}\right) \in K_{t} \quad \text { such that } \Pi\left(\xi_{t}\right)=\inf _{\chi \in K_{t}} \Pi(\chi)
$$

where $K_{t}=\left\{\chi=(W, w) \in H\left(\Omega_{\gamma}\right)|[W] \nu \geqslant|\left[\frac{\partial w}{\partial \nu}\right] \mid\right.$ on $\left.\gamma ;\left.\quad \chi\right|_{\omega_{t}} \in R\left(\omega_{t}\right)\right\}$. The problem (6) is known to have a unique solution $\xi_{t} \in K_{t}$, which satisfies the variational inequality [21,26].

$$
\xi_{t} \in K_{t}, \quad B\left(\xi_{t}, \chi-\xi_{t}\right) \geqslant \int_{\Omega_{\gamma}} F\left(\chi-\xi_{t}\right) d x \quad \forall \chi \in K_{t}
$$

In parallel with the equilibrium problem for the plate with the volume rigid inclusion we consider also an equilibrium problem for an elastic plate with a delaminated thin flat inclusion. Here we assume that the thin rigid inclusion is described by the cylindrical surface $x=\left(x_{1}, x_{2}\right) \in \gamma$, $-1 \leqslant z \leqslant 1$, while the crack is still located on the positive side $\left(\gamma^{+}\right)$of the inclusion's surface. Let us first introduce some notation:

$$
R(\gamma)=\left\{\zeta(x)=(\rho, l) \mid \rho(x)=b\left(x_{2},-x_{1}\right)+\left(c_{1}, c_{2}\right) ; l(x)=a_{0}+a_{1} x_{1}+a_{2} x_{2}, x \in \gamma\right\},
$$

where $b, c_{1}, c_{2}, a_{0}, a_{1}, a_{2} \in \mathbf{R}$. Unlike previous models (see, for example, [16, 19, 20, 23]), for rigid inclusions in a Kirchhoff-Love plate described by cylindrical surfaces, we require that for $\chi=(W, w)$ the following relations hold:

$$
\chi \in R(\gamma) \text { for } w=a_{0}+a_{1} x_{1}+a_{2} x_{2} \text { conditions } \frac{\partial w^{-}}{\partial x_{1}}=a_{1}, \quad \frac{\partial w^{-}}{\partial x_{2}}=a_{2} \text { on } \gamma .
$$

It is important to note that the last two equalities in (9) are complementary to the condition $\chi \in R(\gamma)$, which was used in $[16,19,20,23]$. These equalities mean that rotation angles of the normal fibers of the plate are equal to some constant values within the entire plane rigid inclusion in the equilibrium state. Therefore, in the case of thin flat inclusion we introduce the following set of admissible functions

$$
K_{0}=\left\{\chi=(W, w) \in H\left(\Omega_{\gamma}\right)|[W] \nu \geqslant|\left[\frac{\partial w}{\partial \nu}\right] \mid, \frac{\partial w^{-}}{\partial x_{i}}=a_{i} \text { on } \gamma ; i=1,2,\left.\chi\right|_{\gamma^{-}} \in R(\gamma)\right\}
$$

where $w=a_{0}+a_{1} x_{1}+a_{2} x_{2}$ on $\gamma^{-}$. Consider a variational formulation of the problem. We want to find a function $\xi_{0}=\left(U_{0}, u_{0}\right) \in K_{0}$, such that

$$
\Pi\left(\xi_{0}\right)=\inf _{\chi \in K_{0}} \Pi(\chi) .
$$

For the new kind of mathematical description of flat rigid inclusions, the variational setting (10) is new. 
We will establish the existence of a solution to the problem in accordance with the Weierstrass theorem [27]. It is well known that the energy functional has the properties of coercivity and weak semicontinuity [21]. Obviously, the set $K_{0}$ is convex. Let us prove the closedness of $K_{0}$. Let us suppose that $\chi_{n} \rightarrow \chi$ strongly in $H\left(\Omega_{\gamma}\right)$ as $n \rightarrow \infty, \chi_{n} \in K_{0}$. In this case, the traces $w_{n} \rightarrow w, \frac{\partial w_{n}}{\partial x_{i}} \rightarrow \frac{\partial w}{\partial x_{i}}$ converge in $L_{2}(\gamma), i=1,2$, and therefore there exists a subsequence still denoted by $n$, such that

$$
w_{n}=a_{0 n}+a_{1 n} x_{1}+a_{2 n} x_{2}, \quad \frac{\partial w_{n}}{\partial x_{i}}=a_{i n} \quad \text { on } \quad \gamma^{-} .
$$

converging a.e. on $\gamma$ to functions $w^{-}, \frac{\partial w^{-}}{\partial x_{i}}, i=1,2$, respectively. Since $\frac{\partial w_{n}}{\partial x_{i}}=a_{i n}$ a. e. on $\gamma^{-}, i=1,2$, we have that $\left\{a_{i n}\right\}, i=1,2$, converge to the some numbers $\hat{a}_{i}, i=1,2$. Therefore, $\frac{\partial w^{-}}{\partial x_{i}}=\hat{a}_{i}, i=1,2$. Next, in view of convergence

$$
w_{n}^{-}=a_{0 n}+a_{1 n} x_{1}+a_{2 n} x_{2} \rightarrow w \quad \text { a.e. on } \quad \gamma
$$

we get

$$
\lim _{n \rightarrow \infty} a_{0 n}=w^{-}-\hat{a}_{1} x_{1}+\hat{a}_{2} x_{2} \quad \text { a. e. on } \gamma .
$$

Finally, there exists a limit $\lim _{n \rightarrow \infty} a_{0 n}=\hat{a}_{0}$ and $w=\hat{a}_{0}+\hat{a}_{1} x_{1}+\hat{a}_{2} x_{2}$ a. e. on $\gamma^{-}$. The fulfillment of the relations (3) for the limit function can be established by standard methods, see [21]. So, $\chi$ belongs to $K_{0}$ and the set is closed. For the problem (10), the conditions of the Weierstrass theorem on the solvability of the problem are satisfied. It is easy to show that its solution $\xi_{0} \in K_{0}$ will be unique and satisfies the following variational inequality [24]

$$
\xi_{0} \in K_{0}, \quad B\left(\xi_{0}, \chi-\xi_{0}\right) \geqslant \int_{\Omega_{\gamma}} F\left(\chi-\xi_{0}\right) d x \quad \forall \chi \in K_{0} .
$$

\section{An optimal control problem}

Consider the cost functional

$$
J(t)=\left\|\xi_{t}-\xi^{*}\right\|_{H\left(\Omega_{\gamma}\right)}, \quad t \in\left[0, t_{0}\right],
$$

where $\xi^{*} \in H\left(\Omega_{\gamma}\right)$ is a prescribed element, $\xi_{t}$ is the solution of the problem (6) for $t>0$ and $\xi_{0}$ is the solution of the problem (10). We have to find a solution of the maximization problem

$$
\sup _{t \in\left[0, t_{0}\right]} J(t)
$$

The following assertion holds.

Theorem 2.1. There exists a solution of the optimal control problem (12).

Proof. Let $\left\{t_{n}\right\}$ be a maximizing sequence. By the boundedness of the segment $\left[0, t_{0}\right]$, we can extract a convergent subsequence $\left\{t_{n_{k}}\right\} \subset\left\{t_{n}\right\}$ such that

$$
t_{n_{k}} \rightarrow t^{*} \quad \text { as } \quad k \rightarrow \infty, \quad t^{*} \in\left[0, t_{0}\right]
$$

Without loss of generality we assume that $t_{n_{k}} \neq t^{*}$ for sufficiently large $k$. Otherwise there would exist a sequence $\left\{t_{n_{l}}\right\}$ such that $t_{n_{l}} \equiv t^{*}$, and therefore the value $t^{*}$ is solution of (12). Consider 
the case of the subsequence $\left\{t_{n_{k}}\right\}$ satisfying $t_{n_{k}} \neq t^{*}$ for sufficiently large $k$. Now we take into account Lemma 2, proved below: the solutions $\xi_{k}$ of (6) corresponding to the parameters $t_{n_{k}}$ converge to the solution $\xi_{t^{*}}$ strongly in $H\left(\Omega_{\gamma}\right)$ as $k \rightarrow \infty$. This allows us to obtain convergence

$$
J\left(t_{n_{k}}\right) \rightarrow J\left(t^{*}\right)
$$

This means that

$$
J\left(t^{*}\right)=\sup _{t \in\left[0, t_{0}\right]} J(t)
$$

The theorem is proved.

Before proceeding further we first prove the following lemma.

Lemma 1. Let $t^{*} \in\left[0, t_{0}\right]$ be a fixed real number and let $\left\{t_{n}\right\} \subset\left[t^{*}, t_{0}\right]$ be a sequence of real numbers converging to $t^{*}$ as $n \rightarrow \infty$. Then for an arbitrary function $\eta=(V, v) \in K_{t^{*}}$ there exist a subsequence $\left\{t_{k}\right\}=\left\{t_{n_{k}}\right\} \subset\left\{t_{n}\right\}$ and a sequence of functions $\left\{\eta_{k}\right\}$ such that $\eta_{k}=\left(V_{k}, v_{k}\right) \in K_{t_{k}}$, $k \in \mathbf{N}$ and $\eta_{k} \rightarrow \eta$ weakly in $H\left(\Omega_{\gamma}\right)$ as $k \rightarrow \infty$.

Proof. If there exists a subsequence $\left\{t_{n_{k}}\right\}$ such that $t_{n_{k}}=t^{*}$, then the assertion of this lemma holds for the sequence $\eta_{k} \equiv \eta, k \in \mathbf{N}$. Therefore, below we assume that $t_{n}>t^{*}$ for sufficiently large $n$. Denote by $\zeta^{*}=\left(\rho^{*}, l^{*}\right)$ with $\rho^{*}=b^{*}\left(x_{2},-x_{1}\right)+\left(c_{1}^{*}, c_{2}^{*}\right), l^{*}(x)=a_{0}^{*}+a_{1}^{*} x_{1}+a_{2}^{*} x_{2}$ the function describing the structure of $\eta$ in $\omega_{t}$ for the case $t^{*}>0$. If $t^{*}=0$ and the function $\eta$ has the specified structure on $\gamma^{-}$, then adopt the same notation, i.e. $\zeta^{*}=\eta$ on $\gamma^{-}$. We extend the definition of $\zeta^{*}=\left(\rho^{*}, l^{*}\right)$ to the whole domain $\Omega$ by the equalities:

$$
\zeta^{*}=\left(\rho^{*}, l^{*}\right), \quad \text { where } \begin{cases}\rho^{*}=b^{*}\left(x_{2},-x_{1}\right)+\left(c_{1}^{*}, c_{2}^{*}\right), & x \in \Omega, \\ l^{*}(x)=a_{0}^{*}+a_{1}^{*} x_{1}+a_{2}^{*} x_{2}, & x \in \Omega .\end{cases}
$$

Fix an arbitrary value $t \in\left(0, t_{0}\right]$ and consider the following family of auxiliary problems.

$$
\text { Find } \quad \eta_{t} \in K_{t}^{\prime} \quad \text { such that } p\left(\eta_{t}\right)=\inf _{\chi \in K_{t}^{\prime}} p(\chi)
$$

where $p(\chi)=\|\chi-\eta\|^{2}$

$$
K_{t}^{\prime}=\left\{\chi=(W, w) \in H\left(\Omega_{\gamma}\right) \mid \chi=\eta, w_{\nu}=v_{\nu} \text { on } \gamma^{ \pm},\left.\quad \chi\right|_{\omega_{t}}=\zeta^{*}\right\} .
$$

It is easy to see that the functional $p(\chi)$ is coercive and weakly lower semicontinuous on the space $H\left(\Omega_{\gamma}\right)$. It can be verified that the set $K_{t}^{\prime}$ is convex and closed in $H\left(\Omega_{\gamma}\right)$. This properties guarantee the existence of a solution of the problem (13). Besides, the solution $\eta_{t}$ is unique [24,27].

Since the functional $p(\chi)$ is convex and differentiable on $H\left(\Omega_{\gamma}\right)$, the problem (13) can be written in the equivalent form:

$$
\eta_{t} \in K_{t}^{\prime}, \quad\left(\eta_{t}-\eta, \chi-\eta_{t}\right)_{H\left(\Omega_{\gamma}\right)} \geqslant 0 \quad \forall \chi \in K_{t}^{\prime}
$$

where $\left(\eta_{t}-\eta, \chi-\eta_{t}\right)_{H\left(\Omega_{\gamma}\right)}$ is a scalar product in $H\left(\Omega_{\gamma}\right)$. By the property $\left.\mathbf{b}\right)$, it is evident that the solution $\eta_{t_{0}}$ of $(14)$ for $t=t_{0}$ belongs to the set $K_{t}^{\prime}$ with $t^{\prime} \in\left(0, t_{0}\right]$. Substituting $\eta_{t_{0}}$ as the test functions into (14), we get

$$
\left(\eta_{t}-\eta, \eta_{t_{0}}\right)_{H\left(\Omega_{\gamma}\right)}+\left(\eta, \eta_{t}\right)_{H\left(\Omega_{\gamma}\right)} \geqslant\left(\eta_{t}, \eta_{t}\right)_{H\left(\Omega_{\gamma}\right)} \quad \forall t \in\left(0, t_{0}\right]
$$

Using the inequality (2) we obtain from this relation the following uniform upper bound:

$$
\left\|\eta_{t}\right\| \leqslant c \quad \forall t \in\left(0, t_{0}\right]
$$


Therefore, we can extract from the sequence $\left\{\eta_{t_{n}}\right\}$ a subsequence $\left\{\eta_{k}\right\}$, which is defined by equalities $\eta_{k}=\eta_{t_{n_{k}}}, k \in \mathbf{N}$ (henceforth we define a sequence $\left\{t_{k}\right\}$ by the equality $t_{k}=t_{n_{k}}$ ) and $\left\{\eta_{k}\right\}$ weakly converges to some function $\widetilde{\eta}$ in $H\left(\Omega_{\gamma}\right)$.

Show that $\widetilde{\eta}=\eta$. To this end we must distinguish two cases for $t^{*}$, namely $t^{*}>0$ and $t^{*}=0$. Let us first assume that $t^{*}>0$. Then, by construction $\left(\eta_{k}-\eta\right) \in H_{0}^{1}\left(\Omega_{\gamma} \backslash \bar{\omega}_{t^{*}}\right)^{2} \times H_{0}^{2}\left(\Omega_{\gamma} \backslash \bar{\omega}_{t^{*}}\right)$. Consequently, in virtue of the weak closedness of $H_{0}^{1}\left(\Omega_{\gamma} \backslash \bar{\omega}_{t^{*}}\right)^{2} \times H_{0}^{2}\left(\Omega_{\gamma} \backslash \bar{\omega}_{t^{*}}\right)$ we have $(\widetilde{\eta}-\eta) \in$ $H_{0}^{1}\left(\Omega_{\gamma} \backslash \bar{\omega}_{t^{*}}\right)^{2} \times H_{0}^{2}\left(\Omega_{\gamma} \backslash \bar{\omega}_{t^{*}}\right)$. We consider now the functions of the form $\chi_{k}^{ \pm}=\eta_{k} \pm \phi$, where $\phi \in C_{0}^{\infty}\left(\Omega_{\gamma} \backslash \bar{\omega}_{t^{*}}\right)^{3} \cap C_{0}^{\infty}\left(\Omega_{\gamma}\right)^{3}$. Bearing in mind the property $\left.\mathbf{c}\right)$, observe that $\chi_{k}^{ \pm} \in K_{t_{k}}$ for sufficiently large $k$. We next substitute the elements of these sequences $\left\{\chi_{k}^{+}\right\}$and $\left\{\chi_{k}^{-}\right\}$as test functions into inequalities (14) corresponding to $t_{k}$. As a result, we obtain

$$
\eta_{k} \in K_{t_{k}}^{\prime}, \quad\left(\eta_{k}-\eta, \phi\right)_{H\left(\Omega_{\gamma}\right)}=0
$$

Fix the function $\phi$. Passing to the limit in (15), we deduce

$$
(\widetilde{\eta}-\eta, \phi)_{H\left(\Omega_{\gamma}\right)}=0 \quad \forall \phi \in C_{0}^{\infty}\left(\Omega_{\gamma} \backslash \bar{\omega}_{t^{*}}\right)^{3} \cap C_{0}^{\infty}\left(\Omega_{\gamma}\right)^{3} .
$$

Hence, by the density of $C_{0}^{\infty}\left(\Omega_{\gamma} \backslash \bar{\omega}_{t^{*}}\right)$ both in $H_{0}^{1}\left(\Omega_{\gamma} \backslash \bar{\omega}_{t^{*}}\right)$ and $H_{0}^{2}\left(\Omega_{\gamma} \backslash \bar{\omega}_{t^{*}}\right)$, we infer that $\widetilde{\eta}-\eta=$ 0 in $H_{0}^{1}\left(\Omega_{\gamma} \backslash \bar{\omega}_{t^{*}}\right)^{2} \times H_{0}^{2}\left(\Omega_{\gamma} \backslash \bar{\omega}_{t^{*}}\right)$. Finally, by construction, the equality $\widetilde{\eta}=\eta$ is fulfilled in $\omega_{t^{*}}$. Therefore, $\widetilde{\eta}=\eta$ in $H\left(\Omega_{\gamma}\right)$ and there is a sequence $\left\{\eta_{k}\right\}$ such that $\eta_{k} \in K_{t_{k}}, k \in \mathbf{N}$ and $\eta_{k} \rightarrow \eta$ weakly in $H\left(\Omega_{\gamma}\right)$ as $n \rightarrow \infty$.

Let us consider the second case. Suppose that $t^{*}=0$. By construction, we have $\left(\eta_{k}-\eta\right) \in$ $H_{0}^{1}\left(\Omega_{\gamma}\right)^{2} \times H_{0}^{2}\left(\Omega_{\gamma}\right)$, and consequently, the relation $(\widetilde{\eta}-\eta) \in H_{0}^{1}\left(\Omega_{\gamma}\right)^{2} \times H_{0}^{2}\left(\Omega_{\gamma}\right)$ is fulfilled. We now consider functions of the form $\chi_{k}^{ \pm}=\eta_{k} \pm \phi$, where $\phi \in C_{0}^{\infty}\left(\Omega_{\gamma}\right)^{3}$. Observe that the property d) yields that for sufficiently large $k$ the inclusion $\chi_{k}^{ \pm} \in K_{t_{k}}$ holds. Substituting these functions in (14) corresponding to $t_{k}$, yields the equality

$$
\eta_{k} \in K_{t_{k}}^{\prime}, \quad\left(\eta_{k}-\eta, \phi\right)_{H\left(\Omega_{\gamma}\right)}=0
$$

We fix the function $\phi$ in (16) and pass to the limit as $k \rightarrow \infty$. As a result, we get

$$
(\widetilde{\eta}-\eta, \phi)_{H\left(\Omega_{\gamma}\right)}=0 \quad \forall \phi \in C_{0}^{\infty}\left(\Omega_{\gamma}\right)^{3} .
$$

The density of $C_{0}^{\infty}\left(\Omega_{\gamma}\right)$ in $H_{0}^{n}\left(\Omega_{\gamma}\right)(n \in \mathbf{N})$ allows us to obtain from (17) the equality $\widetilde{\eta}-\eta=0$ in $H_{0}^{1}\left(\Omega_{\gamma}\right)^{2} \times H_{0}^{2}\left(\Omega_{\gamma}\right)$. It remains to observe that $\widetilde{\eta}=\eta$ on $\gamma^{ \pm}$by the construction. Thus, $\widetilde{\eta}=\eta$ in $H\left(\Omega_{\gamma}\right)$ and there exists a subsequence of functions $\left\{\eta_{k}\right\}$ such that $\eta_{k} \in K_{t_{k}}$ and $\eta_{k} \rightarrow \eta$ weakly in $H\left(\Omega_{\gamma}\right)$. The lemma is proved.

\section{Limiting passage by the parameter of the transverse thickness of inclusions}

The following result, in particular, allows us to assert that the problem (10) is the limit for problems of the form (6). It establishes a continuous dependence of solutions on the parameter of the transverse thickness of the inclusions.

Lemma 2. Let $t^{*} \in\left[0, t_{0}\right]$ be a fixed real number. Then $\xi_{t} \rightarrow \xi_{t^{*}}$ strongly in $H\left(\Omega_{\gamma}\right)$ as $t \rightarrow t^{*}$, where $\xi_{t}$ is the solution of $(6)$ corresponding to $t \in\left(0, t_{0}\right]$ while $\xi_{t^{*}}$ is the solution corresponding to (6) for $t^{*}>0$ and to the problem (10) for $t^{*}=0$. 
Proof. We will prove it by contradiction. Let us assume that there exist a number $\epsilon_{0}>0$ and a sequence $\left\{t_{n}\right\} \subset\left(0, t_{0}\right]$ such that $t_{n} \rightarrow t^{*},\left\|\xi_{n}-\xi_{t^{*}}\right\| \geqslant \epsilon_{0}$, where $\xi_{n}=\xi_{t_{n}}, n \in \mathbf{N}$ are the solutions of (6) corresponding to $t_{n}$.

Since $\chi^{0} \equiv 0 \in K_{t}$ for all $t \in\left[0, t_{0}\right]$, we can substitute $\chi=\chi^{0}$ in (7) for all $t \in\left(0, t_{0}\right]$ and in (11) for $t=0$. This provides

$$
\xi_{t} \in K_{t}, \quad B\left(\xi_{t}, \xi_{t}\right) \leqslant \int_{\Omega_{\gamma}} F \xi_{t} d x, \quad \forall t \in\left[0, t_{0}\right] .
$$

From here, using (2) we can deduce that for all $t \in\left[0, t_{0}\right]$ the following estimate holds

$$
\left\|\xi_{t}\right\| \leqslant c
$$

with some constant $c>0$ independent of $t$. Consequently, replacing $\xi_{n}$ with a subsequence if necessary, we can assume that $\xi_{n}$ converges to some $\tilde{\xi}$ weakly in $H\left(\Omega_{\gamma}\right)$.

Now we show that $\tilde{\xi} \in K_{t^{*}}$. Indeed, we have $\left.\xi_{n}\right|_{\omega_{t_{n}}}=\zeta_{n} \in R\left(\omega_{t_{n}}\right)$. In accordance with the Sobolev embedding theorem [28], we obtain that

$$
\begin{aligned}
&\left.\xi_{n}\right|_{\omega_{t^{*}}}\left.\rightarrow \tilde{\xi}\right|_{\omega_{t^{*}}} \quad \text { strongly in } L_{2}\left(\omega_{t^{*}}\right)^{3} \text { as } n \rightarrow \infty, \\
&\left.U_{n}\right|_{\gamma}\left.\rightarrow \tilde{U}\right|_{\gamma} \quad \text { strongly in } L_{2}(\gamma)^{2} \text { as } n \rightarrow \infty, \\
&\left.\left.\frac{\partial u_{n}}{\partial x_{i}}\right|_{\gamma} \rightarrow \frac{\partial \tilde{u}}{\partial x_{i}}\right|_{\gamma} \quad \text { strongly in } L_{2}(\gamma) \text { as } n \rightarrow \infty .
\end{aligned}
$$

Choosing a subsequence, if necessary, we assume as $n \rightarrow \infty$ that $\xi_{n} \rightarrow \tilde{\xi}$ a.e. in $\omega_{t^{*}}$. This allows us to conclude that each of the numerical sequences $\left\{b_{n}\right\},\left\{c_{1 n}\right\},\left\{c_{2 n}\right\},\left\{a_{0 n}\right\},\left\{a_{1 n}\right\},\left\{a_{2 n}\right\}$ defining the structure of $\zeta_{n}$ in domains $\omega_{t_{n}}$ is bounded in absolute value. Thus, we can extract subsequences (retain notation) such that

$$
b_{n} \rightarrow b, a_{0 n} \rightarrow a_{0}, c_{i n} \rightarrow c_{i}, a_{i n} \rightarrow a_{i}, i=1,2, \text { as } n \rightarrow \infty .
$$

Further we must distinguish two different cases: $t^{*}=0$ and $t^{*}>0$. In the first case for the sequence $\left\{\xi_{n}\right\}$ corresponding to the specified convergent number sequences $\left\{b_{n}\right\},\left\{c_{1 n}\right\},\left\{c_{2 n}\right\}$, $\left\{a_{0 n}\right\},\left\{a_{1 n}\right\},\left\{a_{2 n}\right\}$ we have

$$
\begin{aligned}
& \left.U_{n}\right|_{\gamma^{-}} \rightarrow b\left(x_{2},-x_{1}\right)+\left(c_{1}, c_{2}\right) \text { strongly in } L_{2}(\gamma)^{2} \text { as } n \rightarrow \infty \text {, } \\
& \left.u_{n}\right|_{\gamma^{-}} \rightarrow a_{0}+a_{1} x_{1}+a_{2} x_{2} \text { strongly in } L_{2}(\gamma) \text { as } n \rightarrow \infty \text {, } \\
& \left.\frac{\partial u_{n}}{\partial x_{i}}\right|_{\gamma^{-}} \rightarrow a_{i} \text { strongly in } L_{2}(\gamma) \text { as } n \rightarrow \infty, \quad i=1,2 .
\end{aligned}
$$

The last three relations with (18) leads to the equality

$$
\left.\tilde{U}\right|_{\gamma^{-}}=b\left(x_{2},-x_{1}\right)+\left(c_{1}, c_{2}\right),\left.\quad \tilde{u}\right|_{\gamma^{-}}=a_{0}+a_{1} x_{1}+a_{2} x_{2} \quad \text { a. e. on } \gamma \text {. }
$$

This means $\left.\tilde{\xi}\right|_{\gamma^{-}} \in R(\gamma)$ and

$$
\left.\frac{\partial \tilde{u}}{\partial x_{i}}\right|_{\gamma^{-}}=a_{i} \quad \text { a. e. on } \gamma, \quad i=1,2 .
$$

Consider the second case. If there exists a subsequence $\left\{t_{k}\right\} \subset\left\{t_{n}\right\}$ such that $t_{k} \geqslant t^{*}$ for all $k \in \mathbf{N}$, then we can easily obtain the following convergences

$$
\left.U_{k}\right|_{\omega_{t^{*}}} \rightarrow b\left(x_{2},-x_{1}\right)+\left(c_{1}, c_{2}\right) \text { strongly in } L_{2}\left(\omega_{t^{*}}\right)^{2} \text { as } k \rightarrow \infty,
$$




$$
\left.u_{k}\right|_{\omega_{t^{*}}} \rightarrow a_{0}+a_{1} x_{1}+a_{2} x_{2} \text { strongly in } L_{2}\left(\omega_{t^{*}}\right) \text { as } k \rightarrow \infty .
$$

Therefore, from (21), (22) and (18) we obtain $\left.\tilde{\xi}\right|_{\omega_{t^{*}}} \in R\left(\omega_{t^{*}}\right)$.

Suppose that there exists a subsequence $\left\{t_{k}\right\} \subset\left\{t_{n}\right\}$ satisfying $t_{k}<t^{*}$ for all $k \in \mathbf{N}$ and $t_{k} \rightarrow t^{*}$ as $k \rightarrow \infty$. In this case for an arbitrary fixed $k^{\prime} \in \mathbf{N}$ and the corresponding value $t^{\prime}=t_{k^{\prime}}$, by the property $\mathbf{b}$ ) we have

$$
\begin{array}{r}
U_{k} \rightarrow b\left(x_{2},-x_{1}\right)+\left(c_{1}, c_{2}\right) \text { strongly in } L_{2}\left(\omega_{t^{\prime}}\right)^{2} \text { as } k \rightarrow \infty, \\
u_{k} \rightarrow a_{0}+a_{1} x_{1}+a_{2} x_{2} \text { strongly in } L_{2}\left(\omega_{t^{\prime}}\right) \text { as } k \rightarrow \infty .
\end{array}
$$

It is possible to define a function $l=a_{0}+a_{1} x_{1}+a_{2} x_{2}$ in $\omega_{t^{*}}$. In view of the absolute continuity of the Lebesgue integral and the properties $\mathbf{b}$ ) and $\mathbf{f}$ ), for any $\epsilon>0$ we can choose a number $k^{\prime} \in \mathbf{N}$ large enough such that

$$
\|l\|_{L^{2}\left(\omega_{t^{*}} \backslash \omega_{t^{\prime}}\right)}<\sqrt{\epsilon}, \quad\|\tilde{u}\|_{L^{2}\left(\omega_{t^{*}} \backslash \omega_{t^{\prime}}\right)}<\sqrt{\epsilon} .
$$

Further, using the triangle inequality, it follows from this that

$$
\begin{aligned}
& \left\|u_{k}-l\right\|_{L^{2}\left(\omega_{t^{*}} \backslash \omega_{t^{\prime}}\right)} \leqslant\left\|u_{k}\right\|_{L^{2}\left(\omega_{t^{*}} \mid \omega_{t^{\prime}}\right)}+\|l\|_{L^{2}\left(\omega_{t^{*}} \backslash \omega_{t^{\prime}}\right)} \leqslant
\end{aligned}
$$

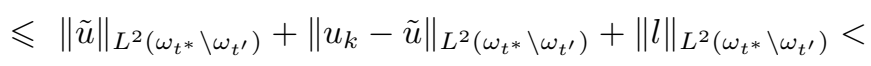

$$
\begin{aligned}
& <2 \sqrt{\epsilon}+\left\|u_{k}-\tilde{u}\right\|_{L^{2}\left(\omega_{t^{*}}\right)} \text {. }
\end{aligned}
$$

Therefore we have

$$
\begin{aligned}
\left\|u_{k}-l\right\|_{L^{2}\left(\omega_{t^{*}}\right)}^{2}=\left\|u_{k}-l\right\|_{L^{2}\left(\omega_{t^{*}} \backslash \omega_{t^{\prime}}\right)}^{2}+ & \left\|u_{k}-l\right\|_{L^{2}\left(\omega_{t^{\prime}}\right)}^{2}< \\
& <\left(2 \sqrt{\epsilon}+\left\|u_{k}-\tilde{u}\right\|_{L^{2}\left(\omega_{t^{*}}\right)}\right)^{2}+\left\|u_{k}-l\right\|_{L^{2}\left(\omega_{t^{\prime}}\right)}^{2} .
\end{aligned}
$$

We can see that for all sufficiently large numbers $k$ we have the following estimates

$$
\left\|u_{k}-\tilde{u}\right\|_{L^{2}\left(\omega_{t^{*}}\right)}<\sqrt{\epsilon}, \quad\left\|u_{k}-l\right\|_{L^{2}\left(\omega_{t^{\prime}}\right)}<\sqrt{\epsilon}
$$

and the right-hand side of (23) is less than $10 \epsilon$. Therefore, $u_{k} \rightarrow l$ strongly in $L^{2}\left(\omega_{t^{*}}\right)$. Consequently, taking into account (18) we get $\left.\tilde{u}\right|_{\omega_{t^{*}}}=l$ in $\omega_{t^{*}}$.

It can be proved analogously that $\left.\tilde{U}\right|_{\omega_{t^{*}}}=b\left(x_{2},-x_{1}\right)+\left(c_{1}, c_{2}\right)$ a.e. in $\omega_{t^{*}}$. Thus, we conclude that $\left.\tilde{\xi}\right|_{\omega_{t^{*}}} \in R\left(\omega_{t^{*}}\right)$. Therefore, in all possible cases we have $\left.\tilde{\xi}\right|_{\omega_{t^{*}}} \in R\left(\omega_{t^{*}}\right)$.

It remains to show that $\tilde{\xi}$ satisfies the inequality

$$
\left[\frac{\partial \tilde{u}}{\partial \nu}\right] \geqslant 0, \quad[\tilde{U}] \nu \geqslant\left[\frac{\partial \tilde{u}}{\partial \nu}\right], \quad[\tilde{u}]=0 \text { on } \gamma .
$$

In view of (19), (20), we can extract subsequences once again and obtain the following convergences $\left.\left.\xi_{n}\right|_{\gamma} \rightarrow \tilde{\xi}\right|_{\gamma},\left.\left.\frac{\partial u_{n}}{\partial \nu}\right|_{\gamma} \rightarrow \frac{\partial \tilde{u}}{\partial \nu}\right|_{\gamma}$ a.e. on both $\gamma^{+}$and $\gamma^{-}$. Now we pass to the limit in the following inequalities as $n \rightarrow \infty$

$$
\left[\frac{\partial u_{n}}{\partial \nu}\right] \geqslant 0, \quad\left[U_{n}\right] \nu \geqslant\left[\frac{\partial u_{n}}{\partial \nu}\right], \quad\left[u_{n}\right]=0 \text { on } \gamma .
$$

As a result of passing to the limit, we get (24) and $\tilde{\xi} \in K_{t^{*}}$.

Observe that, as $t_{n} \rightarrow t^{*}$, there must exist either a subsequence $\left\{t_{n_{l}}\right\}$ such that $t_{n_{l}} \leqslant t^{*}$ for all $l \in \mathbf{N}$ or, if that is not the case, a subsequence $\left\{t_{n_{m}}\right\}, t_{n_{m}}>t^{*}$ for all $m \in \mathbf{N}$. For the 
first case we have the subsequence $\left\{t_{n_{l}}\right\} \subset\left(0, t_{0}\right]$ with the property $t_{n_{l}} \leqslant t^{*}$ for all $l \in \mathbf{N}$. This implies that $t^{*}>0$. For convenience, we denote this subsequence by $\left\{t_{n}\right\}$. Since $t_{n} \leqslant t^{*}$, by the property b) the arbitrary test function $\chi \in K_{t^{*}}$ also belongs to the set $K_{t_{n}}$. This property allows us to pass to the limit as $n \rightarrow \infty$ in the following inequalities with the test functions $\chi \in K_{t^{*}}$ :

$$
\xi_{n} \in K_{t_{n}}, \quad B\left(\xi_{n}, \chi-\xi_{n}\right) \geqslant \int_{\Omega_{\gamma}} F\left(\chi-\xi_{n}\right) d x, \quad t_{n} \in\left(0, t^{*}\right]
$$

Taking into account the weak convergence of $\xi_{n}$ to $\tilde{\xi}$, the last inequality in the limit takes the form

$$
B(\tilde{\xi}, \chi-\tilde{\xi}) \geqslant \int_{\Omega_{\gamma}} F(\chi-\tilde{\xi}) d x \quad \forall \chi \in K_{t^{*}}
$$

This means that $\tilde{\xi}=\xi_{t^{*}}$. To complete the proof for the first case we must establish the strong convergence $\xi_{n} \rightarrow \xi_{t^{*}}$. By substituting $\chi=2 \xi_{t}$ and $\chi=0$ into the variational inequalities (7) for $t \in\left(0, t_{0}\right]$, we get

$$
\xi_{t} \in K_{t}, \quad B\left(\xi_{t}, \xi_{t}\right)=\int_{\Omega_{\gamma}} F \xi_{t} d x \quad \forall t \in\left(0, t_{0}\right]
$$

In view of (7) this means that the following relation

$$
\xi_{t} \in K_{t}, \quad B\left(\xi_{t}, \chi\right) \geqslant \int_{\Omega_{\gamma}} F \chi d x \quad \forall \chi \in K_{t}
$$

holds for all $t \in\left(0, t_{0}\right]$. Hence, by the weak convergence $\xi_{n} \rightarrow \xi_{t^{*}}$ in $H\left(\Omega_{\gamma}\right)$ as $n \rightarrow \infty$, we deduce

$$
\lim _{n \rightarrow \infty} B\left(\xi_{n}, \xi_{n}\right)=\lim _{n \rightarrow \infty} \int_{\Omega_{\gamma}} F \xi_{n} d x=\int_{\Omega_{\gamma}} F \xi_{t^{*}} d x=B\left(\xi_{t^{*}}, \xi_{t^{*}}\right) .
$$

Since we have the equivalence of norms (see Remark 1), one can see that $\xi_{n} \rightarrow \xi_{t^{*}}$ strongly in $H\left(\Omega_{\gamma}\right)$ as $n \rightarrow \infty$. Thus in the first case we get a contradiction to the assumption: $\left\|\xi_{n}-\xi_{t^{*}}\right\| \geqslant \epsilon$ for all $n \in \mathbf{N}$.

Consider the second case, i.e. we suppose that elements of the subsequence $\left\{t_{n_{m}}\right\}$ satisfy $t_{n_{m}}>t^{*}$ for all $m \in \mathbf{N}$. For convenience we keep the same notation for the subsequence. In doing so we have $t_{n} \rightarrow t^{*}$ and $t_{n}>t^{*}$. Taking into account the results at the beginning of the proof, we have that $\xi_{n} \rightarrow \tilde{\xi}$ weakly in $H\left(\Omega_{\gamma}\right)$ as $n \rightarrow \infty$. For instance we will prove that $\xi_{n} \rightarrow \tilde{\xi}$ strongly in $H\left(\Omega_{\gamma}\right)$ as $n \rightarrow \infty$. In view of the weak convergence $\xi_{n} \rightarrow \tilde{\xi}$ in $H\left(\Omega_{\gamma}\right)$ as $n \rightarrow \infty$, from (25) we deduce

$$
\lim _{n \rightarrow \infty} B\left(\xi_{n}, \xi_{n}\right)=\int_{\Omega_{\gamma}} F \tilde{\xi} d x
$$

Next, substituting $\chi=\xi_{t^{\prime}} \in K_{t^{\prime}} \subset K_{t}$, for arbitrary fixed numbers $t, t^{\prime} \in\left(0, t_{0}\right]$ such that $t^{\prime} \geqslant t$, in (26) as the test function, we arrive at the inequality

$$
B\left(\xi_{t}, \xi_{t^{\prime}}\right) \geqslant \int_{\Omega_{\gamma}} F \xi_{t^{\prime}} d x
$$

Therefore, we conclude that for all $t_{n}$ and $t_{m}$ satisfying $t_{n} \leqslant t_{m}$ the following inequality is fulfilled

$$
B\left(\xi_{n}, \xi_{m}\right) \geqslant \int_{\Omega_{\gamma}} F \xi_{m} d x
$$

Fix an arbitrary value $m$ in (28) and pass to the limit in the last relation as $n \rightarrow \infty$. As a result we have

$$
B\left(\tilde{\xi}, \xi_{m}\right) \geqslant \int_{\Omega_{\gamma}} F \xi_{m} d x .
$$


Passing to the limit in (29) as $m \rightarrow \infty$, we find

$$
B(\tilde{\xi}, \tilde{\xi}) \geqslant \int_{\Omega_{\gamma}} F \tilde{\xi} d x
$$

This inequality, the formula (27), and the weak lower semicontinuity of bilinear form $B(\cdot, \cdot)$ yield the following chain of relations

$$
B(\tilde{\xi}, \tilde{\xi}) \geqslant \int_{\Omega_{\gamma}} F \tilde{\xi} d x=\lim _{n \rightarrow \infty} B\left(\xi_{n}, \xi_{n}\right) \geqslant B(\tilde{\xi}, \tilde{\xi}) .
$$

This means that

$$
B(\tilde{\xi}, \tilde{\xi})=\lim _{n \rightarrow \infty} B\left(\xi_{n}, \xi_{n}\right) .
$$

Again, by the equivalence of norms (see Remark 1), we deduce that $\xi_{n} \rightarrow \tilde{\xi}$ strongly in $H\left(\Omega_{\gamma}\right)$ as $n \rightarrow \infty$.

From Lemma 1, for any $\eta \in K_{t^{*}}$ there exist a subsequence $\left\{t_{k}\right\}=\left\{t_{n_{k}}\right\} \subset\left\{t_{n}\right\}$ and a sequence of functions $\left\{\eta_{k}\right\}$ such that $\eta_{k} \in K_{t_{k}}$ and $\eta_{k} \rightarrow \eta$ weakly in $H\left(\Omega_{\gamma}\right)$ as $k \rightarrow \infty$.

The properties established above for the convergent sequences $\left\{\eta_{k}\right\}$ and $\left\{\xi_{n}\right\}$ allow us to pass to the limit as $k \rightarrow \infty$ in following inequalities derived from (7) for $t_{k}$ and with test functions $\eta_{k}$ :

$$
B\left(\xi_{k}, \eta_{k}-\xi_{k}\right) \geqslant \int_{\Omega_{\gamma}} F\left(\eta_{k}-\xi_{k}\right) d x .
$$

As a result, we have

$$
B(\tilde{\xi}, \eta-\tilde{\xi}) \geqslant \int_{\Omega_{\gamma}} F(\eta-\tilde{\xi}) d x \quad \forall \eta \in K_{t^{*}}
$$

The unique solvability of this variational inequality implies that $\tilde{\xi}=\xi_{t^{*}}$. Therefore, in either case there exist a subsequence $\left\{t_{n_{k}}\right\} \subset\left\{t_{n}\right\}$ such that $t_{k} \rightarrow t^{*}, \xi_{k} \rightarrow \xi_{t^{*}}$ strongly in $H\left(\Omega_{\gamma}\right)$, which is a contradiction. Lemma is proved.

Lemmas 1 and 2 establish a qualitative connection between the equilibrium problems for plates with rigid delaminated inclusions of varying transverse thickness. In particular it is shown that as the thickness's parameter $t$ of the volume rigid inclusion tends to zero, the solutions $\xi_{t}$ of the equilibrium problems converge to the solution of the equilibrium problem for the plate containing the flat thin rigid delaminated inclusion.

The first author's work was supported by the Russian Foundation for Basic Research (grant no. 18-29-10007-mk), the 2nd author's work was supported the Ministry of science and higher education of the Russian Federation, supplementary agreement no. 075-02-2020-1543/1, April 29, 2020 .

\section{References}

[1] A.Khludnev, G.Leugering, On elastic bodies with thin rigid inclusions and cracks, Math. Method Appl. Sci., 33(2010), no. 16, 1955-1967.

[2] A.M.Khludnev, M.Negri, Optimal rigid inclusion shapes in elastic bodies with cracks, $Z$. Angew. Math. Phys., 64(2013), no. 1, 179-191. 
[3] F.Dal Corso, D.Bigoni, M.Gei, The stress concentration near a rigid line inclusion in a prestressed, elastic material. Part I, Full-field solution and asymptotics, J. Mech Phys. Solids, 56(2008), no. 3, 815-838.

[4] I.I.Il'ina, V.V.Sil'vestrov, The problem of a thin interfacial inclusion detached from the medium along one side, Mech. Solids., 40(2005), no. 3, 123-133.

[5] H.Itou, A.M.Khludnev, E.M.Rudoy, A.Tani, Asymptotic behaviour at a tip of a rigid line inclusion in linearized elasticity, Z. Angew. Math. Mech., 92(2012), no. 9, 716-730.

[6] E.M.Rudoy, The Griffith formula and Cherepanov-Rice integral for a plate with a rigid inclusion and a crack, J. Math. Sci. 186(2012), no. 3, 511-529.

[7] Z.M.Xiao, B.J.Chen, Stress intensity factor for a Griffith crack interacting with a coated inclusion, Int. J. Fract., 108(2001), no. 3, 193-205.

[8] R.V.Namm, G.I.Tsoy, Solution of a contact elasticity problem with a rigid inclusion. Comput. Math. and Math. Phys. 59(2019), 659-666. DOI: 10.1134/S0965542519040134

[9] E.Rudoy, On numerical solving a rigid inclusions problem in 2D elasticity, Z. Angew. Math. Phys., 68(2017), N. 19. DOI: 10.1007/s00033-016-0764-6.

[10] N.Lazarev, H.Itou, Optimal location of a rigid inclusion in equilibrium problems for inhomogeneous Kirchhoff-Love plates with a crack, Math. Mech. Solids, 24(2019), no. 12, 3743-3752. DOI: $10.1177 / 1081286519850608$

[11] N.Lazarev, G.Semenova, An optimal size of a rigid thin stiffener reinforcing an elastic twodimensional body on the outer edge, J. Optim. Theory Appl. 178(2018), 614-626.

DOI: $10.1007 / \mathrm{s} 10957-018-1291-8$.

[12] N.P.Lazarev, H.Itou, N.V.Neustroeva, Fictitious domain method for an equilibrium problem of the Timoshenko-type plate with a crack crossing the external boundary at zero angle, Jpn. J. Ind. Appl. Math., 33(2016), no. 1, 63-80.

[13] N.V.Neustroeva, A rigid inclusion in the contact problem for elastic plates, J. Appl. Indust. Math., 4(2010), no. 4, 526-538.

[14] E.M.Rudoy, Shape derivative of the energy functional in a problem for a thin rigid inclusion in an elastic body, Z. Angew. Math. Phys., 66(2014), no. 4, 1923-1937.

[15] A.M.Khludnev, Optimal control of crack growth in elastic body with inclusions, Eur. J. Mech. A Solids, 29(2010) no. 3, 392-399.

[16] V.V.Shcherbakov, Existence of an optimal shape of the thin rigid inclusions in the KirchhoffLove plate, J. Appl. Indust. Math., 8(2014), no. 1, 97-105.

[17] V.V.Shcherbakov, Choosing an optimal shape of thin rigid inclusions in elastic bodies, $J$. Appl. Mech. Tech. Phy., 56(2015), 321-329.

[18] I.V. Frankina, A contact problem for an elastic plate with a thin rigid inclusion, J. Appl. Industr. Math., 10(2016), no. 3, 333-340. DOI: 10.17377/SIBJIM.2016.19.309 
[19] A.M.Khludnev, On bending an elastic plate with a delaminated thin rigid inclusion, J. Appl. Indust. Math. 5(2011), no. 4, 582-594.

[20] N.Lazarev, Existence of an optimal size of a delaminated rigid inclusion embedded in the Kirchhoff-Love plate, Bound Value Probl., (2015). DOI: 10.1186/s13661-015-0437-y.

[21] A.M.Khludnev, Elasticity problems in nonsmooth domains, Moscow, Fizmatlit, 2010 (in Russian).

[22] A.M.Khludnev, A.A.Novonty, J.Sokolowsky, A.Zochowski, Shape and topology sensitivity analysis for cracks in elastic bodies on boundaries of rigid inclusions, J. Mech. Phys. Solids, 57(2009), no. 10, 1718-1732.

[23] A.M.Khludnev, Shape control of thin rigid inclusions and cracks in elastic bodies, Arch. Appl. Mech. 83(2013), no. 10, 1493-1509.

[24] A.M.Khludnev, V.A.Kovtunenko, Analysis of cracks in solids, Southampton, WIT-Press, 2000.

[25] N.P.Lazarev, V.V.Everstov, N.A.Romanova, Fictitious domain method for equilibrium problems of the Kirchhoff-Love plates with nonpenetration conditions for known configurations of plate edges, Journal of Siberian Federal University Mathematics and Physics, 12(2019), no. 6, 674-686. DOI: 10.17516/1997-1397-2019-12-6-674-686.

[26] A.M.Khludnev, Problem of a crack on the boundary of a rigid inclusion in an elastic plate, Mech. Solids, 45(2010), no. 5, 733-742.

[27] C.Baiocchi, A.Capello, Variational and Quasivariational Inequalities: Application to free boundary problems, New York, Wiley, 1984.

[28] V.G.Maz'ja, Sobolev Spaces, Springer Series in Soviet Mathematics, Berlin, 1985.

\title{
О предельном переходе по толщине жесткого включения в задаче о равновесии пластины Кирхгофа-Лява с трещиной
}

\author{
Нюргун П. Лазарев \\ Галина М. Семенова \\ Наталья А. Романова \\ Северо-Восточный федеральный университет \\ Якутск, Российская Федерация
}

\begin{abstract}
Аннотация. В работе рассмотрены модели о равновесии пластин с жесткими включениями двух видов. Первый вид включения описывается трехмерным множеством, второй вид жесткого включения соответствует плоскому жесткому включению, которое в исходном состоянии перпендикулярно срединной плоскости. Для обеих моделей вдоль части жесткого включения расположена сквозная трещина. На трещине задаются условия непроникания для случая известной конфигурации изгиба вблизи трещины. Доказана однозначная разрешимость новой постановки задачи для пластины с плоским жестким включением. Доказано, что предельный переход в семействе вариационных задач для пластин с включением первого вида при стремлении параметра поперечной толщины включения к нулю доставляет задачу для пластины с плоским жестким включением. Доказана разрешимость задачи оптимального управления размером жесткого включения.
\end{abstract}

Ключевые слова: вариационная задача, трещина, предельный переход, условие непроникания, задача оптимального управления. 